\title{
Joint Channel Estimation and Prediction for OFDM Systems
}

\author{
Ian C. Wong and Brian L. Evans \\ Wireless Networking and Communications Group \\ Dept. of Electrical and Computer Engineering \\ 1 University Station C0803 \\ The University of Texas at Austin, Austin, Texas 78712 \\ Email: $\{$ iwong, bevans $\} @$ ece.utexas.edu
}

\begin{abstract}
Adaptive OFDM improves the system throughput by adjusting transmission parameters based on channel state information (CSI) estimated at or received by the transmitter. The improvement, however, is conditioned on the quality of the CSI, which can be compromised by estimation and quantization errors, and more significantly by delay. Channel prediction has been previously proposed to combat feedback delay. In this paper, a novel OFDM channel prediction algorithm that uses a $2 \times$ 1 -dimensional frequency estimation to determine the time-delays and Doppler frequencies of each propagation path is investigated. The algorithm assumes a general far-field scatterer, frequencyselective wireless channel model and hence is applicable to a wide variety of wireless channel conditions. It is shown that the method requires less feedback information and has better meansquared error performance than previous methods. We provide simulation results for IEEE 802.16e channels.
\end{abstract}

\section{INTRODUCTION}

Adaptive OFDM systems overcome the limitation of conventional OFDM by allowing the transmitter to vary the power, modulation, and coding on each subcarrier depending on the current channel state information (CSI) [1].This requires the transmitter to have knowledge of the CSI, which can be obtained through feedback from the receiver's channel estimates, or through its own estimates in a time division duplex (TDD) reciprocal channel. In high mobility environments, where the Doppler frequency is high and the channel changes rapidly, the CSI used by the transmitter would be outdated due to the processing and feedback delays.

In [1], delayed CSI was shown to negatively impact the capacity and bit error rate of the adaptive OFDM system. Furthermore, it was shown that the use of channel prediction can improve the performance of the system. In [2], channel prediction over a longer range was shown to improve the performance of adaptive OFDM in a low-mobility environment. In [3], decision-directed and adaptive short-term channel prediction on the time-domain channel taps was proposed. In [4], an unbiased channel power predictor was applied to the time-domain channel taps, and a preliminary evaluation of frequency domain channel prediction on all the subcarriers was also presented.

In this paper, we predict OFDM channels by using classical estimation principles. These principles assume that the parameters being estimated are unknown deterministic quantities.

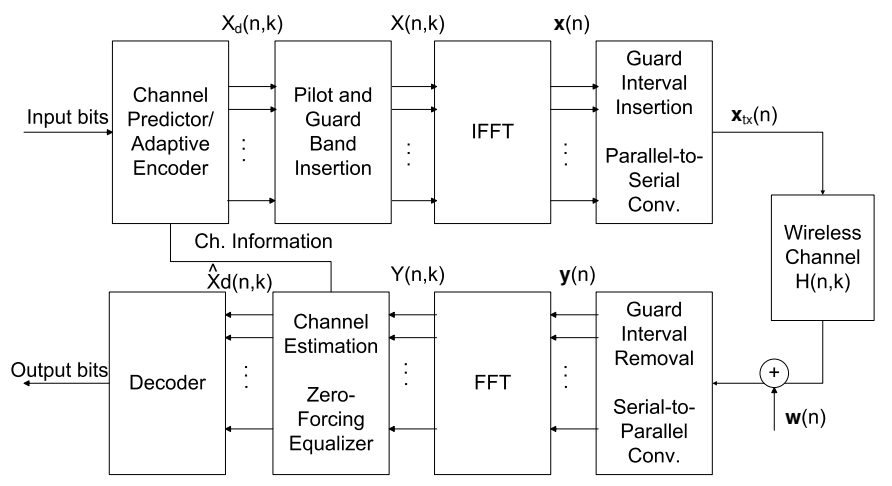

Fig. 1. Adaptive OFDM System Block Diagram

We employ a general far-field scatterer, frequency-selective wireless channel model [5], which models each propagation path as a complex exponential with unknown Doppler frequency, time delay, and complex amplitude. A simplified $2 \times 1$ dimensional frequency and amplitude estimation algorithm that accounts for the specialized structure in the wireless channel is proposed. The algorithm has the advantage of producing reliable channel estimates, and thus is a joint OFDM estimation and prediction solution. The proposed method can be applied to a wide variety of wireless channel conditions, and requires less feedback information and has better prediction performance than previously proposed methods.

\section{SySTEM MODEL}

\section{A. OFDM System}

The adaptive OFDM system model considered in this paper is given in Fig. 1. The input bits are initially mapped by a bank of adaptive encoders into $N_{\text {data }}$ complex data symbols $X_{\text {data }}(n, k)$ which corresponds to the $k$ th subcarrier in the $n$th OFDM block. The constellation density for each encoder would depend on the predicted state of the wireless channel, in which various bit and power allocation strategies may be used to either maximize the data rate or to minimize the power given a bit error rate (BER) constraint [6]. 
The combination of data, pilot, and guard symbols form the $N$-subcarrier OFDM symbol $X(n, k)$. This is subsequently transformed into a time domain sequence using the $N$-point IFFT. In order to avoid intersymbol interference (ISI), and allow the output sequence to be effectively circularly convolved with the channel, the last $\nu$ samples of the symbol samples $\left\{x_{i}(n)\right\}_{i=1}^{N}$ are used as a guard interval (GI) and prepended to the block to form the transmit sequence $x_{i, t x}(n)=x_{l}(n)$, where $l=((i+N-\nu) \oplus N)$ for $i=0,1, \cdots, N+\nu-1$ and $\oplus$ is the modulo operator. It is assumed that $\nu$ is longer than the delay spread of the channel, and that the effects of inter-carrier interference can be ignored; hence, the received signal for the $k$ th subcarrier in the $n$th OFDM block can be written as $Y(n, k)=H(n, k) X(n, k)+W(n, k)$ where $H(n, k)$ and $W(n, k)$ are frequency domain channel gain and additive white Gaussian noise (AWGN) respectively. The channel estimation block takes $Y(n, k)$ as input and forms the channel estimates $\hat{H}(n, k)$ to detect the transmitted sequence as $\hat{X}_{d a t a}(n, k)=Y(n, k) / \hat{H}(n, k)$.

In [2] [3] [4], channel estimates are fed back to the transmitter to enable prediction. In our method, only the estimated channel parameters are fed back in order for the channel prediction block to generate the predicted channel estimates $\hat{H}(n+\Delta, k), \Delta=1, \ldots, \xi$ where $\xi$ is the number of steps ahead to predict.

\section{B. Wireless Channel Model}

The complex baseband representation of the time-varying wireless channel is given as [5]

$$
h_{c}(t, \eta)=\sum_{p=0}^{L-1} \gamma_{p}(t) \delta\left(\eta-\eta_{p}(t)\right)
$$

where $\eta_{p}(t)$ is the delay, $\gamma_{p}(t)$ is the complex amplitude of the $p$ th multipath tap, and $L$ is the number of propagation paths. Assuming a far-field discrete scatterer model, $\gamma_{p}(t)$ can be further decomposed as [5]

$$
\gamma_{p}(t)=\sum_{r=0}^{M_{p}-1} a_{r, p} e^{j 2 \pi \nu_{r, p}(t) t}
$$

where $M_{p}$ is the number of rays contributing to the $p$ th path, and $a_{r, p}$ and $\nu_{r, p}(t)$ are the complex amplitude and Doppler frequency, respectively, for the $r$ th ray in the $p$ th path. Note that the random phase from the complex exponentials have been incorporated into $a_{r, p}$. Also note that the time delays and Doppler frequencies are all dependent on time. However, we can assume that the time delay $\eta_{p}(t)$ and Doppler frequency $\nu_{r, p}(t)$ parameters vary slowly when compared to the OFDM symbol time, and can be considered constant within the estimation and prediction time horizons. Tracking algorithms can also be employed in order to follow the time variations of these parameters [7], but these are not pursued in this work.

Combining (1) and (2) and taking its Fourier transform, we get the frequency response of the time-varying channel as

$$
H_{c}(t, f)=\sum_{p=0}^{L-1} \sum_{r=0}^{M_{p}-1} a_{r, p} e^{j 2 \pi \nu_{r, p} t} e^{-j 2 \pi \eta_{p} f}
$$

Assuming that the OFDM system with symbol period $T_{\text {sym }}$ and subcarrier spacing $\Delta f$ have proper cyclic extension and sample timing, the sampled channel frequency response at the $k$ th tone of the $n$th OFDM block can be expressed as

$$
\begin{aligned}
H(n, k) & \triangleq H_{c}\left(n T_{s y m}, k \Delta f\right) \\
& =\sum_{p=0}^{L-1} \sum_{r=0}^{M_{p}-1} a_{r, p} e^{j 2 \pi\left(f_{r, p} n-\tau_{p} k\right)}
\end{aligned}
$$

where $f_{r, p}=\nu_{r, p} T_{\text {sym }}$ is the normalized Doppler frequency and $\tau_{p}=\eta_{p} \Delta f$ is the normalized time delay.

\section{OFDM CHANNEL PREDICTION AlgorithM}

We assume a pilot-assisted OFDM system, in which a block of $N_{\text {block }}$ pilot symbols is indexed in time by the set $\mathcal{N}=$ $\left\{n_{i} \mid i=1, \ldots, N_{\text {block }}\right\}$ and spaced $\Delta_{t}$ apart to be used for channel estimation. In each OFDM pilot symbol, let $\mathcal{K}=$ $\left\{k_{j} \mid j=1, \ldots, N_{\text {pilot }}\right\}$ denote the set of pilot indices spaced $\Delta_{f}$ apart. We can then perform a least-squares estimate of the channel at the pilot subcarriers using the received signal $Y\left(n_{i}, k_{j}\right)$ and the known pilot symbols $X_{p}\left(n_{i}, k_{j}\right)$ as

$$
\begin{aligned}
\hat{H}\left(n_{i}, k_{j}\right) & =Y\left(n_{i}, k_{j}\right) / X_{p}\left(n_{i}, k_{j}\right) \\
& =H\left(n_{i}, k_{j}\right)+W\left(n_{i}, k_{j}\right) / X_{p}\left(n_{i}, k_{j}\right) \\
& =\sum_{p=0}^{L-1} \sum_{r=0}^{M_{p}-1} a_{r, p} e^{j 2 \pi\left(f_{r, p} n_{i}-\tau_{p} k_{j}\right)}+\widetilde{W}\left(n_{i}, k_{j}\right)
\end{aligned}
$$

For notational convenience, let

$$
\hat{\mathbf{H}}_{L S}=\left[\begin{array}{l|l|l|l}
\hat{\mathbf{h}}_{1}^{1: N_{\text {pilot }}} \mid & \hat{\mathbf{h}}_{2}^{1: N_{\text {pilot }}} \mid & \ldots & \hat{\mathbf{h}}_{N_{\text {block }}}^{1: N_{\text {pilot }}}
\end{array}\right]
$$

be the $N_{\text {pilot }} \times N_{\text {block }}$ matrix of the least-squares estimates, where

$$
\hat{\mathbf{h}}_{i}^{l: m}=\left[\begin{array}{llll}
\hat{H}\left(n_{i}, k_{l}\right) & \hat{H}\left(n_{i}, k_{l+1}\right) & \ldots & \hat{H}\left(n_{i}, k_{m}\right)
\end{array}\right]^{T}
$$

is the column vector of the estimates on time index $n_{i}$ and pilot indices $\left\{k_{j}\right\}_{j=l}^{m}$.

The initial task for the channel predictor is to determine the unknown parameters $L, M_{p}, a_{r, p}, f_{r, p}$, and $\tau_{p}$ from the least squares estimates reliably. Since these terms are assumed to be stationary throughout our estimation and prediction horizon, the next step would be to simply plug in the future time-index to our model and generate the predicted channel. Estimating the parameters in (5) is in the form of a two-dimensional complex sum-of-sinusoids in white noise parameter estimation, and is quite well studied in the literature for radar and sonar imaging and other array signal processing applications (see [8] and references therein). Although a straightforward application of these techniques may be used, they are too computationally intensive for cost-effective online implementation, and do not exploit the special structure of the problem. 
We propose using a $2 \times 1$-dimensional sinusoidal parameter estimation approach. Notice that we can rewrite (5) as

$$
\hat{H}\left(n_{i}, k_{j}\right)=\sum_{p=0}^{L-1} c_{p}\left(n_{i}\right) e^{j 2 \pi \tau_{p} k_{j}}+\widetilde{W}\left(n_{i}, k_{j}\right)
$$

where

$$
c_{p}\left(n_{i}\right)=\sum_{r=0}^{M_{p}-1} a_{r, p} e^{j 2 \pi f_{r, p} n_{i}}
$$

is the complex gain for the $p$ th propagation path ${ }^{1}$, and is the sampled version of (2).

Notice that (8) is now a much simpler one-dimensional complex sum-of-sinusoids parameter estimation, where various standard iterative and non-iterative algorithms have been proposed in the literature (see [9] and the references therein). In this type of estimation problem, the difficulty lies in the estimation of the frequencies of the complex exponentials ( $\tau_{p}$ in our case), since these frequencies enter the model in a non-linear fashion. Once these frequencies are estimated, the complex amplitudes $c_{p}\left(n_{i}\right)$ are easily computed as a linear regression. Although the maximum-likelihood estimates for the frequencies are desirable, techniques to find these exactly or even approximately are highly complex iterative procedures that are not guaranteed to converge [9]. Hence, we opted to base our algorithm on the non-iterative eigen-analysis technique Estimation of Signal Parameters via Rotational Invariance Techniques (ESPRIT) as described in [9]. We discuss the steps in estimating the parameters below.

\section{A. Estimate the autocorrelation matrix}

In order to use eigen-analysis based techniques, the autocorrelation function of the underlying process has to be estimated. Since there are $N_{\text {block }}$ OFDM symbols, we can use an average of the frequency autocorrelation estimates for each symbol generated using the modified covariance method [9],

$$
\hat{\mathbf{R}}^{f}=\frac{1}{N_{\text {block }}} \sum_{i=1}^{N_{\text {block }}} \frac{1}{2}\left(\hat{\mathbf{\Psi}}\left(n_{i}\right)+\mathbf{J} \hat{\mathbf{\Psi}}\left(n_{i}\right)^{H} \mathbf{J}\right)
$$

where

$$
\hat{\mathbf{\Psi}}\left(n_{i}\right)=\frac{1}{N_{\text {pilot }}} \sum_{j=K}^{N_{\text {pilot }}}\left(\hat{\mathbf{h}}_{i}^{(j-K+1): j}\right)\left(\hat{\mathbf{h}}_{i}^{(j-K+1): j}\right)^{H}
$$

and $\mathbf{J}$ is the exchange matrix with ones on the anti-diagonal and zeros elsewhere, and $K$ is the size of the autocorrelation matrix chosen to be greater than the maximum possible number of paths ${ }^{2} L_{\max }$ and less than $N_{\text {pilot }}$.

\footnotetext{
${ }^{1}$ Note that this is the common model used for frequency selective fading channels, where $\left\{c_{p}\left(n_{i}\right)\right\}_{p=1}^{L}$ are typical assumed to be uncorrelated widesense stationary narrow-band complex Gaussian processes with Jakes's power spectrum [5]. We do not make this assumption here, and is thus a more general model.

${ }^{2}$ We make the reasonable assumption that $L_{\max }$ is known à priori. This value is typically determined by the propagation environment, and the desired accuracy of the channel characterization.
}

\section{B. Estimate the number of paths $L$}

Estimation of the number of paths $L$ is essentially a modelselection problem, wherein the Minimum Description Length (MDL) is the method most often used due to its consistency [8]. We employ the MDL appropriate for the modified covariance averaging technique [10] given as

$$
\begin{aligned}
\hat{L}=\arg \min _{1 \leq \mu \leq K-1} & -\log \left(\frac{\left(\prod_{k=\mu+1}^{K} \hat{\lambda}_{k}^{f}\right)^{\frac{1}{K-\mu}}}{\frac{1}{K-\mu} \sum_{k=\mu+1}^{K} \hat{\lambda}_{k}^{f}}\right)^{N_{\text {block }}(K-\mu)} \\
& +\frac{1}{4} \mu(2 K-\mu+1) \log N_{\text {block }}
\end{aligned}
$$

where $\left\{\hat{\lambda}_{k}^{f}\right\}_{k=1}^{K}$ are the non-increasingly ordered estimated eigenvalues of the estimated autocorrelation matrix $\hat{\mathbf{R}}^{f}$.

\section{Estimate the time-delays $\left\{\tau_{p}\right\}_{p=1}^{\hat{L}}$}

Given the estimated number of paths $\hat{L}$, we can now find the time delays $\left\{\tau_{p}\right\}_{p=1}^{\hat{L}}$. In [9], the ESPRIT method was shown to perform better than other eigen-analysis techniques for frequency estimation. Thus, this is the method we pursue in our algorithm. Let

$$
\hat{\mathbf{V}}_{1}=\left[\begin{array}{ll}
\mathbf{I}_{K-1} & \mathbf{0}_{K-1}
\end{array}\right] \mathbf{V}
$$

and

$$
\hat{\mathbf{V}}_{2}=\left[\begin{array}{ll}
\mathbf{0}_{K-1} & \mathbf{I}_{K-1}
\end{array}\right] \mathbf{V}
$$

where $\mathbf{I}_{K-1}$ is the $(K-1) \times(K-1)$ identity matrix, $\mathbf{0}$ is a column vector of $K-1$ zeros, and $\hat{\mathbf{V}}=\left[\begin{array}{ll|l}\hat{\mathbf{v}}_{1} \mid & \ldots & \hat{\mathbf{v}}_{\hat{L}}\end{array}\right]$ is a matrix whose columns are the eigenvectors associated with the $\hat{L}$ largest eigenvalues of $\hat{\mathbf{R}}^{f}$. Then

$$
\hat{\tau}_{p}=-\arg \left(\hat{\epsilon}_{p}\right) / D_{f}, \quad p=1, \ldots, \hat{L}
$$

where $\arg (x)$ is the radian phase angle of the complex number $x$, and $\left\{\hat{\epsilon}_{p}\right\}_{p=1}^{\hat{L}}$ are the eigenvalues of the $\hat{L} \times \hat{L}$ matrix

$$
\hat{\boldsymbol{\Phi}}_{\hat{L}}=\left(\hat{\mathbf{V}}_{1}^{H} \hat{\mathbf{V}}_{1}\right)^{-1} \hat{\mathbf{V}}_{1}^{H} \hat{\mathbf{V}}_{2}
$$

\section{Estimate the complex amplitudes $\left\{c_{p}\left(n_{i}\right)\right\}_{p=1}^{\hat{L}}$}

Given the time delay estimates $\left\{\hat{\tau}_{p}\right\}_{p=1}^{\hat{L}}$, the maximumlikelihood estimate for the $\hat{L} \times N_{\text {block }}$ matrix $\hat{\mathbf{C}}$ of complex amplitudes is given by

$$
\hat{\mathbf{C}}=\left(\mathbf{E}^{H} \mathbf{E}\right)^{-1} \mathbf{E}^{H} \hat{\mathbf{H}}_{L S}
$$

where

$$
\begin{gathered}
\hat{\mathbf{C}}=\left[\begin{array}{llll}
\hat{\mathbf{c}}_{1}^{1: N_{\text {block }}} \mid & \ldots & \mid \hat{\mathbf{c}}_{\hat{L}}^{1: N_{\text {block }}}
\end{array}\right]^{T} \\
\hat{\mathbf{c}}_{p}^{q: r}=\left[\begin{array}{llll}
\hat{c}_{p}\left(n_{q}\right) & \hat{c}_{p}\left(n_{q+1}\right) & \ldots & \hat{c}_{p}\left(n_{r}\right)
\end{array}\right]^{T}
\end{gathered}
$$

and

$$
\mathbf{E}=\left[\begin{array}{ccc}
e^{j 2 \pi \hat{\tau}_{1} k_{1}} & \cdots & e^{j 2 \pi \hat{\tau}_{\hat{L}} k_{1}} \\
\vdots & \ddots & \vdots \\
e^{j 2 \pi \hat{\tau}_{1} k_{N_{p i l o t}}} & \cdots & e^{j 2 \pi \hat{\tau}_{\hat{L}} k_{N_{p i l o t}}}
\end{array}\right]
$$

is the $N_{\text {pilot }} \times \hat{L}$ Fourier transform matrix. 
Note that each of the $N_{\text {block }}$ columns of $\hat{\mathbf{C}}$ in (17) is actually the time-domain channel tap estimate for the frequencyselective channel. Hence, we can write

$$
\hat{\mathbf{H}}_{M L}=\mathbf{W} \hat{\mathbf{C}}
$$

where the $N_{\text {data }} \times N_{\text {block }}$ matrix $\hat{\mathbf{H}}_{M L}$ is the maximumlikelihood estimate for the frequency response of the channel at the $N_{\text {data }}$ data carrying subcarriers, and the $N_{\text {data }} \times \hat{L}$ Fourier transform matrix is given as

$$
\mathbf{W}=\left[\begin{array}{ccc}
e^{j 2 \pi \hat{\tau}_{1}\left(\frac{-N d}{2}\right)} & \cdots & e^{j 2 \pi \hat{\tau}_{\hat{L}}\left(\frac{-N d}{2}\right)} \\
\vdots & \ddots & \vdots \\
e^{j 2 \pi \hat{\tau}_{1}\left(\frac{N d}{2}+1\right)} & \cdots & e^{j 2 \pi \hat{\tau}_{\hat{L}}\left(\frac{N d}{2}+1\right)}
\end{array}\right]
$$

Using (21) as the channel estimates, demodulation of the OFDM symbol can then be performed. Note that minimum mean squared error estimates (MMSE) may also be generated when we assume that the channel is wide-sense-stationary with uncorrelated scatterers (WSSUS), and this is similar to the parametric channel estimation algorithm proposed in [7].

\section{E. Estimate $M_{p}, f_{r, p}$, and $a_{r, p}$}

Notice that we can now replace the left-hand side of (9) $c_{p}\left(n_{i}\right)$ with its corresponding estimate $\hat{c}_{p}\left(n_{i}\right)$, and proceed similarly as the previous steps.

For each path $p=1, \ldots, \hat{L}$,

- Estimate the autocorrelation function $\hat{\mathbf{R}}_{p}^{t}$ across time as in III-A, but removing the averaging in (10), and replacing $\hat{\mathbf{\Psi}}\left(n_{i}\right)$ with

$$
\hat{\mathbf{\Psi}}(p)=\frac{1}{N_{\text {block }}} \sum_{i=I}^{N_{\text {block }}}\left(\hat{\mathbf{c}}_{p}^{(i-I+1): i}\right)\left(\hat{\mathbf{c}}_{p}^{(i-I+1): i}\right)^{H}
$$

- Estimate $M_{p}$ with the MDL using the non-increasing eigenvalues of $\hat{\mathbf{R}}_{p}^{t}$, which is (12) with the $\hat{L}, K$, and the constant in the penalty term of $1 / 4$, replaced by $\hat{M}_{p}$, $I$, and $1 / 2$ respectively.

- Estimate $\left\{f_{r, p}\right\}_{r=1}^{\hat{M}_{p}}$ as in Section III-C, by replacing $\hat{L}$ by $\hat{M}_{p}$ and $K$ by $I$ in (13)-(16).

- Estimate $\hat{\mathbf{a}}_{\mathbf{p}}=\left[\hat{a}_{1, p} \ldots \hat{a}_{\hat{M}_{p}, p}\right]^{T}$ as in III-D, given as

$$
\hat{\mathbf{a}}_{\mathbf{p}}=\left(\mathbf{E}_{t}^{H} \mathbf{E}_{t}\right)^{-1} \mathbf{E}_{t}^{H} \hat{\mathbf{c}}_{\mathbf{p}}
$$

where

$$
\mathbf{E}_{t}=\left[\begin{array}{ccc}
e^{j 2 \pi \hat{f}_{1, p} n_{1}} & \cdots & e^{j 2 \pi \hat{f}_{\hat{M_{p}}} n_{1}} \\
\vdots & \ddots & \vdots \\
e^{j 2 \pi \hat{f}_{1, p} n_{N_{b l o c k}}} & \cdots & e^{j 2 \pi \hat{f}_{\hat{M}_{p}} n_{N_{\text {block }}}}
\end{array}\right]
$$

\section{F. Predict the channel}

Now that we have estimated all the parameters needed in our model, we just plug in these parameters into our model in (4) to find our predicted channel as

$$
\hat{H}(n+\Delta, k)=\sum_{p=0}^{\hat{L}-1} \sum_{r=0}^{\hat{M}_{p}-1} \hat{a}_{r, p} e^{j 2 \pi\left(\hat{f}_{r, p}(n+\Delta)-\hat{\tau}_{p} k\right)}
$$

TABLE I

COMPUTATIONAL COMPLEXITY FOR THE PROPOSED ALGORITHM

\begin{tabular}{||l|l||}
\hline Step & Computation \\
\hline \hline Autocorrelation Estimation & $\hat{L} N_{b l o c k} I^{2}$ \\
\hline Estimation of No. of Paths (MDL) & $\hat{L}\left(\frac{4}{3} I^{3}+I^{2}+\hat{M}_{p} I-\hat{M}_{p}^{2}\right)$ \\
\hline Frequency Estimation (ESPRIT) & $\hat{L}\left(2 I \hat{M}_{p}^{2}+\frac{2}{3} \hat{M}_{p}^{3}+2 \hat{M}_{p}^{2}\right)$ \\
\hline Amplitude Estimation & $\hat{L}\left(2 N_{b l o c k} \hat{M}_{p}^{2}-\frac{2}{3} \hat{M}_{p}^{3}\right)$ \\
\hline Channel Prediction & $\hat{L} \hat{M}_{p} \xi$ \\
\hline
\end{tabular}

TABLE II

COMPUTATIONAL COMPLEXITY FOR BURg PREDICTION

\begin{tabular}{||l|l||}
\hline Step & Computation \\
\hline \hline MDL and coefficients comp. & $\hat{L} 3 N_{\text {block }} p^{2}$ \\
\hline Prediction Filtering & $\hat{L} \xi p$ \\
\hline Interpolation filter design & $\hat{L} \frac{4}{3} B^{3}$ \\
\hline Interpolation & $\hat{L}\left(2 B \Delta_{t}+1\right) \xi$ \\
\hline
\end{tabular}

\section{Performance Comparison}

In comparing the proposed algorithm with previous methods, it has been shown in [11] that prediction on the timedomain channel taps requires less complexity and still performs better than prediction on the subcarriers directly. Thus, we need only to compare our methods with those that predict on the time-domain channel taps [3] [4]. Furthermore, it was shown in [7] that parametric channel estimation performs better than its non-parametric counterpart. Thus, comparing with [3] and [4] directly would give our approach an unfair advantage since their methods use a non-parametric estimator. We compare our algorithm to using standard Wiener linear prediction on the downsampled time-domain channel taps in (18). Furthermore, since the Burg algorithm performs better than the standard Yule-Walker method in deriving the appropriate linear prediction coefficients [2], we used the Burg method in our simulations. We also used the MDL as described in [8] to find the appropriate model orders for the prediction filters. We call this method the Burg Prediction method. We compare the two methods in terms of complexity, required feedback information, and mean-squared error performance.

\section{A. Complexity}

We consider only the computations after the channel estimation step, i.e. Sections III-E and III-F, since this is where the two methods differ. Due to space constraints, we present a summary of the computational complexity analysis in Tables I and II. The details of the Burg Prediction can be found in [2] [11]. The parameter $p$ is the prediction filter order, $2 B \Delta_{t}+1$ is the interpolation filter order, and $\xi$ is the number of steps ahead to predict. Note that in the proposed algorithm, it is the autocorrelation estimation that dominates the required computations, and has complexity $O\left(\hat{L} N_{\text {block }} I^{2}\right)$. For the Burg Prediction, it is the MDL step that dominates, and thus has complexity $O\left(\hat{L} N_{b l o c k} 3 p^{2}\right)$. In our numerical simulations, $I=60$, and the average filter order was $p=35$, thus the complexity of the two methods are comparable. 


\section{B. Required Feedback Information}

In previous methods, the actual channel estimates need to be known by the transmitter. Thus $8 \hat{L} N_{\text {block }}$ bytes must be fed back, assuming each complex channel estimate is represented by two 32-bit single-precision floating point numbers. In the proposed method, only the time-delays $\left\{\hat{\tau}_{p}\right\}_{p=1}^{\hat{L}}$, doppler frequencies $\left\{f_{r, p}\right\}_{r=1, p=1}^{\hat{M}_{p}, \hat{L}_{p}}$, and complex amplitudes $\left\{a_{r, p}\right\}_{r=1, p=1}^{\hat{M}_{p}, \hat{L}_{p}}$ need to be fed back. This amounts to $4 \hat{L}(1+$ $3 \hat{M}_{p}$ ) bytes. Using the simulation parameters below, our proposed method requires only $\frac{1+3 \hat{M}_{p}}{2 N_{\text {block }}} \approx 25 \%$ of feedback required compared to previous methods.

\section{Mean-squared Error}

The OFDM system considered is based on the IEEE 802.16e mobile broadband wireless system [12] in the ETSI "Vehicular A" channel environment (6-tap frequency-selective Rayleigh fading channel model). We simulate the Rayleigh fading channel using the modified Jakes simulator with 32 propagation paths. The OFDM system has bandwidth $B W=5 \mathrm{MHz}$, carrier frequency $f_{c}=2.6 \mathrm{GHz}$, and a Doppler frequency of $f_{d} \approx 180 \mathrm{~Hz}$. A sampling frequency of $f_{s}=\frac{144}{125} \mathrm{BW}=5.76$ $\mathrm{MHz}$, and a guard interval of $N_{g i}=64$ samples is used, giving an OFDM symbol period of $t_{\text {sym }}=\left(N+N_{g i}\right) / f_{s}=55.56 \mu \mathrm{s}$.

In simulating our channel prediction algorithm, we used $N_{\text {pilot }}=26$ pilot subcarriers spaced $\Delta_{f}=8$ subcarriers apart across the middle of the OFDM symbol, and $N_{\text {block }}=100$ OFDM pilot symbols spaced $\Delta_{t}=25$ symbols apart. The autocorrelation matrix size for time-delay estimation in (11) is chosen to be $K=\left\lceil\frac{3}{5} N_{\text {pilot }}\right\rceil=16$, and similarly for the doppler frequency estimation $I=\left\lceil\frac{3}{5} N_{\text {block }}\right\rceil=60$.

We ran our proposed algorithm using ML estimates as in (17) and MMSE estimates as in [7], and compared it with the Burg Prediction method. We generated 100 different channel realizations and 100 iterations for each realization. Figure 2 shows a snapshot of the channel prediction algorithm for the proposed algorithm using ML estimates and Burg Prediction methods for $\mathrm{SNR}=10 \mathrm{~dB}$. It is shown qualitatively that the proposed method predicts the channel better than Burg Prediction. Figure 3 shows the prediction normalized mean square error (NMSE) comparisons for the three different methods predicting $10 t_{c o h}$ ahead for varying SNR (left figure), and predicting in varying lengths for $\mathrm{SNR}=10 \mathrm{~dB}$ (right figure). The proposed methods outperform the Burg Prediction method for all SNR values and prediction lengths, and the advantage is more pronounced as the SNR and the prediction length increase. This is because using the parametric model allows us to focus our estimation on the principal components of the underlying process, and this greatly enhances the prediction performance. This is analogous to the performance improvement brought about by using parametric channel estimation versus non-parametric estimation as observed in [7]. Additionally, the improvement in using the MMSE estimates versus the ML estimates is minimal, and is only seen for lower SNRs.
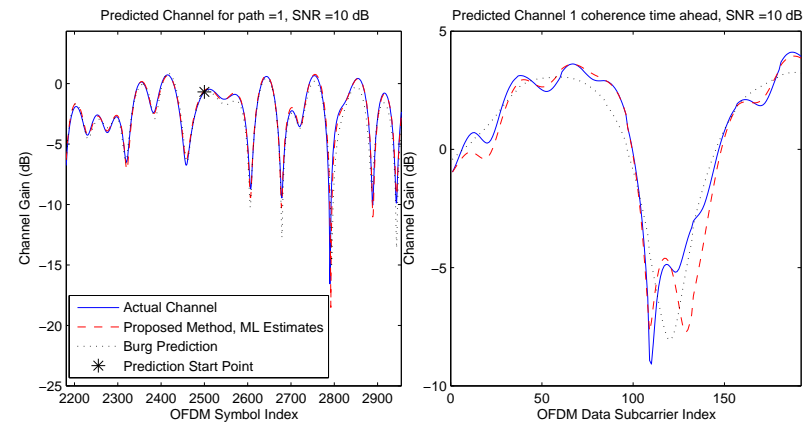

Fig. 2. Snapshot of predicted channel with $\mathrm{SNR}=10 \mathrm{~dB}$. Channel trace for the 1st path is shown on the left, and the frequency domain predicted channel for 1 coherence time ahead is shown on the right.
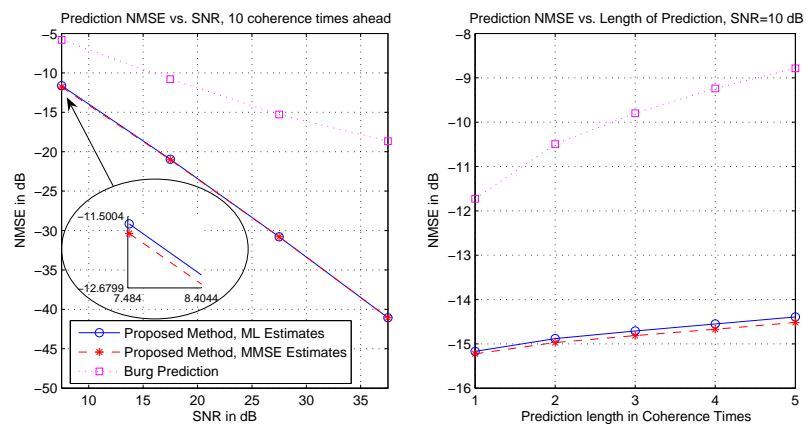

Fig. 3. Normalized mean-squared error (NMSE) performance for the three different methods. The figure on the left shows NMSE vs. SNR, and the figure on the right shows NMSE vs. prediction length.

\section{REFERENCES}

[1] M. R. Souryal and R. L. Pickholtz, "Adaptive Modulation with Imperfect Channel Information in OFDM," in IEEE Proc. Int. Conf. Comm., June 2001, pp. 1861-1865.

[2] A. Forenza, "Exploitation of the Fixed Wireless Channel through the Link-Adaptation Algorithm and the Channel Prediction," Master's thesis, Institut Eurocom, 2001.

[3] D. Schafhuber and G. Matz, "MMSE and Adaptive Prediction of TimeVarying Channels for OFDM Systems," IEEE Trans. Wireless Commun., vol. 4, no. 2, pp. 593-602, Mar. 2005.

[4] M. Sternad and D. Aronsson, "Channel estimation and prediction for adaptive OFDM downlinks," in Proc. IEEE Vehicular Technology Conference, vol. 2, Oct. 2003, pp. 1283-1287.

[5] W. C. Jakes, Microwave Mobile Communications. Wiley, 1974.

[6] T. Keller and L. Hanzo, "Adaptive modulation techniques for duplex OFDM transmission," IEEE Trans. Veh. Technol., vol. 49, no. 5, pp. 1893-1906, Sept. 2000.

[7] B. Yang, K. Letaief, R. Cheng, and Z. Cao, "Channel estimation for OFDM transmission in multipath fading channels based on parametric channel modeling," IEEE Trans. Commun., vol. 49, no. 3, pp. 467-479, Mar. 2001.

[8] S. M. Kay, Modern Spectral Estimation : Theory and Application. Prentice Hall, 1988.

[9] P. Stoica and R. L. Moses, Introduction to Spectral Analysis. Prentice Hall, 1997.

[10] G. Xu, I. R.H. Roy, and T. Kailath, "Detection of number of sources via exploitation of centro-symmetry property," IEEE Trans. Signal Processing, vol. 42, no. 1, pp. 102-112, Jan. 1994.

[11] I. Wong, A. Forenza, R. Heath, and B. Evans, "Long range channel prediction for adaptive OFDM systems," in Proc. IEEE Asilomar Conf. on Signals, Sys. and Comp., vol. 1, CA, Nov. 2004, pp. 732-736.

[12] Air Interface for Fixed and Mobile Broadband Wireless Access Systems, IEEE Std. 802.16e/D5, 2004 